\title{
Embeddedness and sequentiality in social media
}

\author{
Stuart Reeves \\ Mixed Reality Lab, School of Computer Science \\ University of Nottingham, UK \\ stuart@tropic.org.uk
}

\author{
Barry Brown \\ Mobile Life@ Stockholm University \\ SE-164, Kista, Sweden \\ barry@mobilelifecentre.org
}

\begin{abstract}
Over the last decade, there has been an explosion of work around social media within $\mathrm{CSCW}$. A range of perspectives have been applied to the use of social media, which we characterise as aggregate, actor-focussed or a combination. We outline the opportunities for a perspective informed by ethnomethodology and conversation analysis (EMCA) — an orientation that has been influential within CSCW, yet has only rarely been applied to social media use. EMCA approaches can complement existing perspectives through articulating how social media is embedded in the everyday lives of its users and how sequentiality of social media use organises this embeddedness. We draw on a corpus of screen and ambient audio recordings of mobile device use to show how EMCA research is generative for understanding social media through concepts such as adjacency pairs, sequential context, turn allocation / speaker selection, and repair.
\end{abstract}

\section{Author Keywords}

Social media research; social network analysis; ethnomethodology; conversation analysis.

\section{ACM Classification Keywords}

H.5.m. Information interfaces and presentation (e.g., HCI): Miscellaneous.

\section{INTRODUCTION}

Research into social media use has recently become a core part of CSCW, so much so that in 2013 the CSCW conference formally modified its title to include "Social Computing" in recognition of this. Social media represents one of the most significant and widely adopted new expressions of collaborative technology and CSCW has rightly taken on the use of these systems as one of its areas of concern. Fortuitously, the online nature of social media also provides large quantities of readily available data derived from social media systems themselves. Capture and analysis of this data has supported a wide range of

Paste the appropriate copyright/license statement here. ACM now supports three different publication options:

- ACM copyright: ACM holds the copyright on the work. This is the historical approach.

- License: The author(s) retain copyright, but ACM receives an exclusive publication license.

- Open Access: The author(s) wish to pay for the work to be open access. The additional fee must be paid to ACM.

This text field is large enough to hold the appropriate release statement assuming it is single-spaced in Times New Roman 8-point font. Please do not change or modify the size of this text box.

Each submission will be assigned a DOI string to be included here. important insights into the complexities of our online lives.

While this research is diverse, we characterise it as having two core perspectives. The aggregate perspective involves the acquisition of large amounts of data collected directly from social media systems themselves (such as the properties of social networks, or content in terms of 'status updates' or 'tweets') and analyses it to find structures and patterns of aggregate online behaviour. The actor-focussed perspective, in turn, examines smaller scales of data (typically but not exclusively qualitatively) that has been collected from the users of social media themselves, administered via instruments such as surveys and interviews. Social media research also frequently combines these approaches together as a form of triangulation.

In this paper we call for an enrichment of this programme through the development of ethnomethodological and conversation analytic (EMCA) perspectives on social media use. EMCA has been broadly influential for many foundational studies in CSCW, particularly for ethnographic studies of work practice with and around technology. Yet there are few studies of social media which have adopted this perspective (exceptions include [43]). By reviewing concepts from ethnomethodological and conversation analytic orientations, we demonstrate the potential generativity of this perspective for doing social media research. As part of this we unpack how one might go about investigating how social media use comes to be embedded in everyday life through the sequential ordering of action.

The following section expands upon distinctions between aggregate and actor-focussed perspectives. We then outline how an EMCA perspective might take shape, and through this describe concepts of embeddedness and sequentiality. In order to exhibit these empirically, subsequent sections use vignettes from a corpus of screen recordings of mobile device use coupled with ambient audio capture. From this we show how core conversation analytic concerns can illustrate the "ethno-methods" of social media practice.

\section{SOCIAL MEDIA RESEARCH}

In reviewing the literature we restrict ourselves mainly to publications in CSCW and closely related fields. We can distinguish two broad approaches or perspectives taken within this body of work: the aggregate perspective and the actor-focussed perspective. It is important to note that these perspectives are not mutually exclusive and often are combined. Broadly speaking, aggregate perspectives tend to 
examine large-scale patterns in the data, while actorfocussed perspectives tend to be concerned with interpreting user-generated content or responses produced during research interventions.

\section{Aggregate perspectives}

The aggregate perspective uses social media systems themselves as a source of empirical data. This approach documents the structure in mass online behaviour, seeking out patterns that occur across multiple users and uses. It tends to be associated with notions of 'big data', 'data science' and so on. Further, it usually takes a quantitative form, which typically involves processing large amounts of data collected from social media systems, particularly websites and mobile apps such as Twitter, Facebook or Instagram. This data may be collected and aggregated 'from within' the social media system itself (e.g., by mapping friend networks), or it could be derived as an aggregate from logging use (or some combination of both, e.g., Bernstein et al. examined client-side instrumentation of news feed viewing activities alongside Facebook log data [2]). As an aggregate set of data, it is then examined using a range of metrics, often informed by network theory (e.g., tie strength, centrality, density, cliques etc., e.g., [1, 12], but also see [14]). Other approaches take collections of SNSspecific properties such as Facebook 'likes' or 'shares', Twitter 'retweets' or 'hashtags' (e.g., [7]), while others use derived metrics of their own (e.g., 'reputation' [23]) to apply to these aggregate data sets. Sometimes these metrics are combined [54]. As an approach, the aggregate perspective necessarily involves the study of large corpuses of data, so as to produce statistical correlations between different forms of behaviour and thus offer the possibility of producing causal models with predictive qualities [55]. The recent textbook "Analyzing the Social Web" [14] presents many of these methods as a way of following this aggregation approach.

Broadly speaking, the aggregate perspective tends to focus on structural analyses of social media. Examining the structure and form of social media leverages its scale-free, power law features [35]. In particular, analysis of the graph of social relations derives from the work of Watts and social network analysis more widely [59]. The focus of that work has been on the social graph and extracting the mathematical shape or 'form' of the social world, as opposed to actual instances themselves. Yet in practice aggregate perspective also document the content of social media, which in some senses breaks with the theoretical foundations of social network analysis [8]. Studies of content may use more qualitative social research methods that may be similar to the actor-focussed perspective (described below), such as conducting content analysis of the data via various coding strategies (e.g., [24, 19]), bringing with it procedures of inter-rater reliability testing and iterative coding. Yet because of the large volume of data that is rendered collectable by these methods, researchers may also employ a variety of algorithmic coding systems such as sentiment analysis [40] to address scaling problems associated with studying aggregated social media content.

\section{Actor-focussed perspective}

A second distinctive approach takes a more actor-focussed perspective, and is primarily concerned with eliciting users' accounts of their behaviour on social media. So, rather than understand social media as an entity in its own right, the focus is on understanding users' perspectives on their use, and the goals to which social media is put. In this perspective, empirical data is drawn from individual users of social media systems, usually obtained in a way 'external' to the system itself. While actor-focused perspectives are typically qualitative, surveys and rating scales are one quantitative method that is used within actorfocused perspectives (e.g., [28, 10]). In some sense, actorfocussed perspectives are most similar to standard sociological and social psychological research; although large aggregate data sets may be still collected, the success of its findings does not turn fundamentally upon this.

For example, researchers conduct interviews with users (e.g., [27, 58]), ethnographic studies, or collect survey data (e.g., online questionnaires [28], scenario-based surveys [10], etc.). Often this involves recruitment using social media itself (e.g., [58, 37]). Sometimes both interviews and surveys are combined (e.g., [20, 62]). In contrast to aggregate perspectives, the focus is on ways of helping users to recollect upon and review their own social media use, eliciting accounts of their own behaviour with social media systems. Rather than logs or recordings made at the point of use, data is collected after the event. This data may be analysed using a range of standardised coding methods, e.g., axial coding derived from grounded theory, or coding schemes developed from social psychology [28]. Alternatively, more general thematic analysis techniques may be put to use based on a range of different epistemic orientations (e.g., [27]). Broadly speaking the actorfocussed perspective seeks to elicit individual actors' experiences of social media and the uses to which social media is put. There is a predominant focus on the perspective of social media users, their motivations, interpretations and the connections between social media and their other activities.

\section{Combined perspectives}

These two perspectives mirror historic divisions of the structure-agency dichotomies of the social sciences, in which large-scale social structures or individual agency are (alternately) analysed as causal forces in explaining the constitution of society. Yet, in many senses social media research in $\mathrm{CSCW}$ has avoided the intellectual feuds associated with this debate. Instead, the two perspectives are frequently combined, albeit still within a somewhat dichotomous framing. A popular formulation of this is to compare measures derived both from survey data and aggregate social media traces (in terms of e.g., reputation 
[23], social ties [6] or personality [41]). Alternatively the actor-focussed perspective may be employed to elaborate upon data derived from the aggregate perspective. For instance, Gilbert and Karahalios used interviews to further explore metrics like tie strength [12]. In this comparison, often the actor's perspective (i.e., accounts from the user) is subordinated to the authority of data collected from the aggregate perspective. Thus, for instance, one might contrast user 'folk theories' about social media use with traces of behaviour collected online (e.g., [2, 54]).

\section{ETHNOMETHODOLOGY AND CONVERSATION ANALYSIS}

Here, and in the rest of the paper, we attempt to outline a distinctive perspective on studying social media, one that is oriented by ethnomethodological and conversation analytic (EMCA) studies of technology use. This differs in various ways from the two perspectives described above; while it shares some apparent similarities with an actor-focussed perspective, EMCA is concerned with examining how interactional phenomena are made orderly-and therefore meaningful to users - in and through their moment-bymoment activities, as opposed to the post-hoc descriptions from users of those activities. In this way EMCA often makes use of ethnographic techniques and in-the-moment recordings rather than relying on recollection data (which itself would be analysed as a different phenomena-i.e., the work of doing 'a recollection'). We feel that this perspective and emphasis on moment-by-moment, unfolding, real-time human action - although rarely employed for understanding social media-could be generative for enriching social media research.

Ethnomethodology is a familiar social research approach within $\mathrm{CSCW}$. It seeks to explicate the orderly ways in which social interaction is brought off in everyday life [11], and as such it is not concerned with the development of causal explanations of human action (e.g., theory) but rather in articulating the methods that are used by individuals to organising their everyday affairs in the world. Ethnomethodology is concerned with the reflexivity of these methods: i.e., how actions produced by members are done so in ways that make the actions recognisable for other members of the situation. Emerging from the ethnomethodological orientation, conversation analysis [44] focuses strongly on language-in-action, and how the orderliness of talk may be investigated empirically by unpacking the 'machinery' of turn taking, the selection of speakers, the 'repair' of utterances in talk and so on (concepts we will return to later). While the precise relationship between ethnomethodology and conversation analysis is complex [31], we refer to this perspective as 'EMCA' in a broad sense. In various foundational studies of $\mathrm{CSCW}$, it was often EMCA-informed work that pioneered understandings of technology and work practice; writing in 1994, Shapiro argued that EMCA studies had "dominated in CSCW at least since Suchman [i.e., 1987]" [51]. This EMCA-oriented work required close attention to the details of work practice, details that had been mostly absent from the conception of 'work' in previous office information systems research. As Barley and Kunda put it, this focus is on the "moment-by-moment flow of activity" and "the situated integration of tools, documents, action, and interaction". Importantly, researchers looked not just at outcomes or motivations but how things were done in real time, in-the-moment [18]. While the tradition of EMCAoriented studies in CSCW is very much 'alive', there has been an absence of that tradition in offering new perspectives on social media systems specifically.

EMCA offers a phenomenological approach to understanding social media through its central concern for examining human experiences of everyday life (the 'lifeworld') as a practical matter [30]. From an EMCA orientation, the use of social media is fundamentally and inextricably embedded in the continuous temporal flow everyday life. Hence to study social media, we examine how this embeddedness is experienced and accomplished by its users. This means paying more attention to how social media is part and parcel of other locally managed activities. For EMCA, the activities of everyday life are structured in time- some things routinely happen before others. Fundamentally there is a 'sequentiality' to activity, something that has been vital for developing understanding of the orderly nature of talk [44] and bodily interaction [15]. This is a core way in which our lived experiencesi.e., the temporal flow of experience that is encountered by humans as social beings in the world-are made orderly and thus comprehensible, and comprehensible in a shared way, between members of society.

Sequentiality has been pursued strongly by foundational texts in EMCA, such as work that explicates the organisation of turn-taking in talk [45]. Sequentiality has non-trivial implications; Schegloff argues that the organisation of utterances in conversation may be considered a sequence in a "strong fashion" in that "conditional relevance" [46] holds between elements of sequences of talk (i.e., they are not just mere subsequent temporal occurrences) [47]. Hence, utterances in everyday exchanges between conversationalists are constructed by them in various ways that enable other participants to discover when it is 'their turn' and what 'kind' of turn that might be (e.g., whether it is sequentially relevant and adequate for a co-conversationalist to utter "uh huh" in their turn). The preservation of sequentiality has also been a key intellectual resource in CSCW research (e.g., see [38]). Past $\mathrm{CSCW}$ system design has taken account of this, e.g., within online chat environments (e.g., [53, 57]). We believe that revisiting this existing appreciation in $\mathrm{CSCW}$ for the role of sequentiality in ordering online communication could shed new light on social media use. Further, we point to recent conversation analytic studies of social media chat-notably published outside CSCW research - that have uncovered how "participants in both online and spoken interaction are oriented to the same basic contingencies of maintaining 
intersubjectivity and building sequentially organized courses of action" [34] (also see [9]).

\section{METHODOLOGY AND CORPUS}

In order to investigate how an EMCA approach might be taken for studying social media, we repurposed an existing data set of captured mobile device use. The data offered instances of social media use, and let us examine how that use was embedded in the details of users' everyday lives, as well as how it was sequentially organised. Here we describe the origins of the approach, the data set itself, and how the data was collected. It is important to note at this point that we are not using this data set to present the results of a study; instead we are using the vignettes drawn from the data set as generative examples to elaborate a set of key topics for EMCA approaches to studying social media. It should also not be implied that this data or the approach to collecting it is somehow necessary to EMCA-oriented research (e.g., see [9]). Instead we see such data merely as an "aid to the sluggish imagination" (Garfinkel, quoting Herbert Spiegelberg) [11] —one that just so happens to help us observe more clearly the unfolding, moment-by-moment sequentiality of interaction with social media.

\section{Recordings}

Broadly speaking, the data set consists of recordings of screen interactions with smartphones, combined with simultaneously-recorded audio from the device's microphone. This technique enabled us to capture something of the interaction occurring around instances of social media use with the mobile device. The captured audiovisual data was then combined with interviews conducted before and after the collection period, so as to build a diverse corpus of data on social media use as it happens. Fifteen social media users were originally recruited to trial this approach. Screen recording software was installed on their iPhones, which - if not disabled by the participant-captured touch interactions and the display contents when their phone was in use. Each captured set of data was subsequently uploaded to our servers each night. A website was provided for users to go through each clip and highlight clips that they wanted to delete or share with us, but also to add a diary description for each clip of what they were doing. The recording software itself could also be stopped by users or they could choose to delete a recording from the last thirty minutes. Around half the clips in our data corpus have descriptions entered by users. More details of the original dataset are available in prior papers [33]. The data corpus consisted of 3-13 days of phone use per participant, with a median of 7 days per user. This translated into 2,684 video clips of use (over 176 video hours). On average $16.3 \%$ of application launches were of social media apps, with Facebook at 7.6\%, Instagram 5.8\% and Pinterest at $1.3 \%$, and Twitter at $1.10 \%$. Around $15 \%$ of the videos contained some social media application use.

\section{EMBEDDEDNESS OF SOCIAL MEDIA}

As we outlined above, social media use does not take place outside or separately from other activities. It happens in a way that is interwoven and embedded within these other activities in real time. We note that while social media research has indeed explored the role it plays in everyday life in some depth (e.g., [27] and the work of Boyd more broadly [3]), we distinguish our conceptualisation of 'embeddedness' in terms of the primacy that EMCA perspectives give to the real time, continually unfolding aspects of human action.

Our data corpus of recordings, coupled with concurrent capture of talk, gives us unusually detailed access to social media use as it unfolds moment-by-moment. It lets us see (in a limited way) something of the first-hand perspective of the user and the corresponding resources that are available and mobilised so as to help them make sense of, use, and communicate through it. It also moves us away from a reliance upon post-hoc accounts and recollections that we argued tended to be leveraged by the actor-focussed perspective. We can ask questions like: How does social media start being used, stop being used? When it is being used, and how is that usage ordered and integrated into other, parallel activities at the time? Example 1 is a short clip of two (physically co-located) Facebook users (A an B) engaging in a brief conversation about a Facebook post from B. As we join them, A, having seen B's status update ("Whee!"), reformulates it as a question for B.

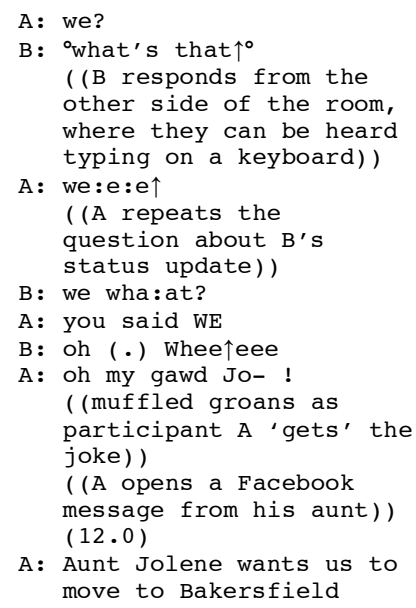

Example 1: Social media as a resource for talk. The extracts make use of Jefferson notation [24] to show some of talk's features such as: [overlapping talk], (pauses), le::ngthened sounds.

Participant A initially does not seem to understand the joke (or, more correctly, participant A produces "we?" which is then followed with "what's that?"). When the "Whee!" is spoken aloud with an elongation of the "e", A gives an amused response. This is perhaps a rather uneventful clip but it is revealing of a number of points.

Firstly, it is worth remarking that an ordinary conversation takes place using, and around, social media. This is recognisably a conversation, and one in which (at least in this case) social media does not distract or disturb the 
conversation as it unfolds. In fact, the short sequence of conversation is occasioned by the Facebook status update. By 'occasioned' we mean that social media makes it possible to have this conversation since it provides the topic of the short exchange. We are reminded here of Sacks's comments on the ways in which 'local resources' (meaning physical objects in the environment) provide topical resources for conversation [44 vol ii p. 92]. What we find here is talk sparked off by something that, once it is found, is common to the conversationalists. Indeed, we were struck by how much co-present social media consumption appeared in the data, with social media offering an array of topical resources to be introduced and discussed. The device then acts as something that can rather naturally be brought into conversation or discarded.

Secondly, an important examinable aspect of B's status update comes from how it is read aloud and discussed in conversation. This is not something that would have been recorded on Facebook itself, and might easily have been missed in recollection. Since the moment-by-moment nature of video gives us some access to real time consumption of social media, it lets us focus our attention on the reading out of the status update. While we only have limited access to this, it is enough to exhibit how Facebook and its 'consumption' are not separate-and thus how Facebook might supply a conversation topic. Indeed, following the status update, a Facebook message is then used to supply the next conversation topic concerning "Aunt Jolene". This leads us to another point. Often social media has been judged as a distraction for social life (as found both popular and academic accounts of interactive technology use e.g., [56]). Yet, when we look at its use embedded in everyday life, it appears instead as expansive; that is, it is generative in occasioning new face to face social interactions around the social media. Collaborative search around phones also exhibits this expansive behaviour-where phone activity can also come to be collaboratively arranged, and so the activity of reading social media becomes something achieved jointly, with 'showings' of the results acting to guide or structure the conversation $[4,42]$.

\section{Temporal projection}

While the previous example exhibited how social media use itself can occasion interaction with others, we can also explore how the use of social media may be arranged in ways that embed this use within the temporal flow of ongoing interaction. In our next example, a Facebook user (C) is browsing videos while he and a group of friends (A and B) are listening to music after dinner (Example 2). C participates in the conversation, but is simultaneously browsing through videos from his Facebook feed. As he waits for Facebook to load more posts, he jumps into conversation. Just after C clicks the "more posts" button in Example 2, he enters into the conversation to make a point about science fiction and fantasy novels.
What we see here is how $\mathrm{C}$ times his interaction with Facebook in concert with the ongoing conversation, pausing in his use during moments where 'hearable' opportunities in talk arise for relevant ways to engage in the conversation (such as C's overlapped response to B's question “don't they just have sci-fi and fantasy books"- the start of which is marked by an arrow in Example 2). Interaction with Facebook here is interwoven with the projected turns-attalk. Hence, $\mathrm{C}$ starts to produce the next turn (breathing in with ".hhhh") at the end of B's turn (i.e., oriented to a transition relevance place) just as Facebook is loading more content. $\mathrm{C}$ also pauses in scrolling through the content until some way into the next speaker's (A) sentence.

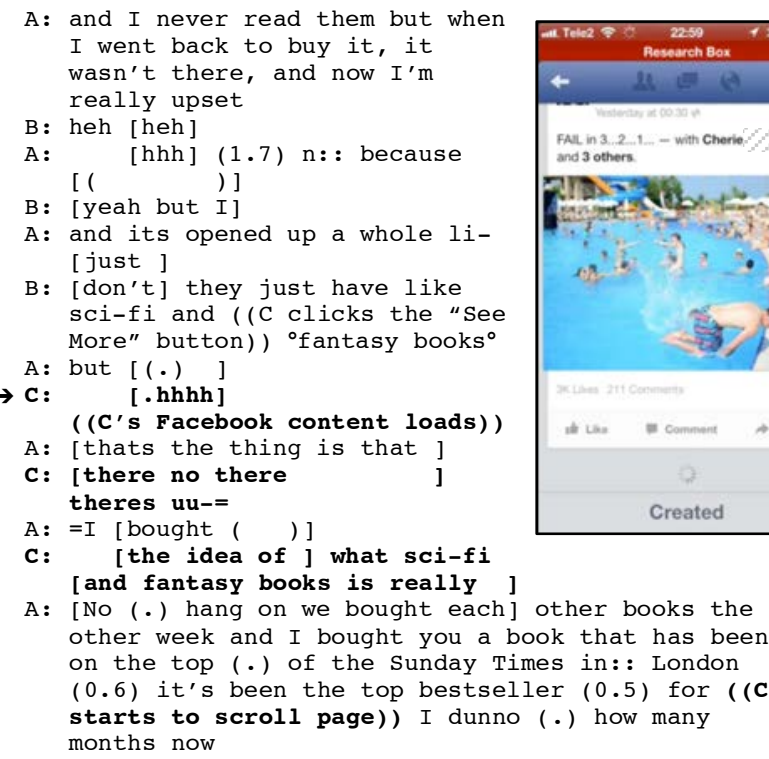

\section{Example 2: Temporal projection}

In other recordings from this session, $\mathrm{C}$ also stops videos when he gets engaged in more focused conversation with a friend, as well as pausing his scrolling at different points depending on the conversation. Licoppe and Figeac describe this as "temporal projection": the decision of what to do with the device relies to an extent on how long the 'gap' that the user has to fill can reasonably expected to be [29]. At the end of our example C starts a conversational turn ("the idea of...", that is cut short by A) while their new content is loading, and then pauses before continuing with looking at more videos.

Temporal projection is not only a matter attended to during conversations. Another clear example of this is when waiting, during which attending to a mobile device may both serve as a way of filling time, but also appear properly engaged in a "minimal main involvement" (in Goffman's terminology) that can be "immediately discarded when one's turn or destination arrives" [13 p. 52]. In an example elsewhere in our data, a participant is meeting a friend at a local square. Location data indicates that the participant arrives in the square, whereupon they check Facebook, 
browsing through item after item for a few minutes. In one view we could gloss these actions as 'killing time'. Yet this description ignores how the sequential order of the actions furnishes them with their meaning. The purpose here is not just to deal with the 'boredom of waiting', but rather about the participant taking advantage of a pause that has opened up through their actions. This is similar to how a conversationalist may take advantage of the projected end of an utterance being completed by a speaker.

In summary, these examples start to offer a way of looking at the embeddedness of social media in the lived experience of its users, as a research topic. We are not claiming we are the first to examine this, but rather underscoring that for EMCA, everyday life becomes a natural first topic of enquiry, in that it forces our attention on the temporally unfolding nature of social media use as it comes to be embedded in the everyday lives of its users. We have set this as a different perspective to looking at aggregate corpora of social media data, or actor-focused recollected accounts from users, valuable though both are. As we move onto our next section we can begin to examine the analytic implications of EMCA's prioritisation of embeddedness, particularly in understanding how social media use is structured sequentially in everyday life.

\section{THE SEQUENTIALITY OF SOCIAL MEDIA}

It is not only that the details of lived experience that are examinable for EMCA. One of its core findings is that this lived experience is orderly and that order can be researched and documented. One of the key aspects of that order is-as we have introduced earlier-sequentiality. As competent members of society, we can use the assumptions of order to recognise and understand what is going on to the point of even reprimanding those involved when sequentiality breaks down. EMCA has documented how sequences are used to order conversation so that we can understand the structure in both our own talk and that of our coconversationalists. Schegloff defines "sequential organization" as "any kind of organization which concerns the relative positioning of utterances or actions $[\ldots]$ turntaking is a type of sequential organization because it concerns the relative ordering of speakers" [50]. Thus, we sought to find sequential organisation in online social media comments and interactions. This is not a hidden order, but an order that is findable for those reading and creating social media content in common.

Critically, sequentiality is not identical to temporal order (in the sense that one thing follows another-indeed, much research on social media use employs the capture of timestamp data), but rather is a method employed by members of society so as to make sense of the experience of temporality. This means that while sequentially-linked events may occur in 'lockstep' with the relentless phenomenon of temporality, as we shall see, there is no 'requirement' that they do so. One of the simplest instances of sequentiality in conversation is an adjacency pair-for example, a question and an answer. These are a 'pair' in that they act together as paired: a question puts a certain prospective compulsion on recipients to answer in some way and thus complete the pair. Questions may not be followed by an answer, of course, but in this case they are seen as unanswered because of the expectation for a 'pair'. Sequentiality also broadly extends to other actions such as walking through a revolving door [60] or playing hopscotch [16]. Sequentiality is has also been documented in various online interactions - text messages [22] and online chat exchanges $[38,21,53]$.

How might sequentiality in conversation help us in understanding social media? After all, social media is a varied and distinct medium. Yet research into online chat $[9,34,38]$, search engine use [36], and text messaging [22] is suggestive of similarities in sequential organisation despite their very different material forms. Although social media use is clearly different to talk, during its developing use, users of social media draw upon their existing practices and methods of interaction. This is somewhat similar to the way conversational practices around phone calls draw upon - and innovate upon - methods employed in ordinary co-present talk, or how use of email draws upon the practices and conventions of letters (although also changed them, such as in the indentation of replies, the addition of complex signatures, and so on). In this section we will examine four sequential organisational forms that we suggest are to be found in social media: adjacency pairs, sequential context, turn allocation / speaker selection, and repair.

\section{Adjacency pairs}

As we have mentioned above, perhaps one of the most prevalent sequential organisational forms in talk is the pair. Besides questions, there are many other adjacency pairs to be found in everyday conversation: greetings, summons and invitations take similar forms. Questions appear in social media frequently; they have been extensively examined as a way for social media users to search for information and maintain network tie relations [17]. Yet, questions on social media are not necessarily about finding out information but also as an action, a way of 'doing things'. So, rather than focusing on questions as information search, we can instead look at their sequential form to see how they are organised as actions in their adjacency pairings, and what gets done in the pairing. In our next example, a user posts a question on Facebook concerning a visit to the movies. Through the comments he receives replies to the message, arranging a trip to the cinema that evening (note that this has been translated from Swedish). 


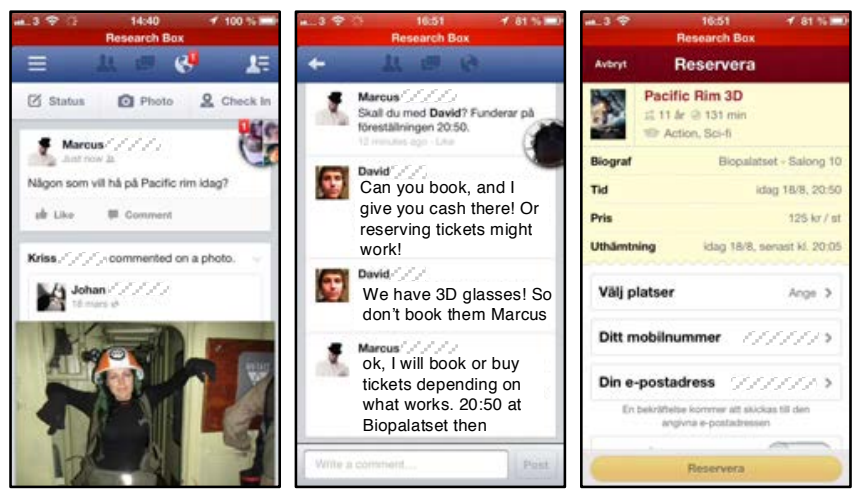

Marcus: Anyone want to see Pacific Rim today?

Philip: Possibly, if I can make the time and if it is not in $3 \mathrm{D}:-$ )

Marcus: The only 2D showing is 17:05

Philip: Little tight possibly. Another time :)

David: Have you booked tickets?

Marcus: I haven't booked

Marcus: Shall we go David? Thinking of 20:50

David: Why not? Can you book and I give you cash there! or reserving tickets might work!

David: We have $3 \mathrm{~d}$ glasses, so don't book them for us.

Marcus: Ok, I will book or buy tickets depending on what works. 20:50 at Bioplatset then

Example 3: Anyone want to see Pacific Rim today?

Clearly the initial question acts as an invitation, i.e., who would want to go to see the movie? The two sets of responses are treated as two second pair-parts to the first question: Philip's "Possibly, if I can make the time and if it is not in 3D" and David's "Have you booked tickets?". Philip's conditional acceptance is followed by some information about the time of the showing, which results in a decline to the initial invitation. As a dispreferred response [39], Philip offers a pre-explanation for the decline ("Little tight possibly"), and declines in the form of an invitation for "another time". However, the reply from David takes the form of another question "Have you booked tickets?", which itself produced a further question "I haven't booked. Should we go David? Thinking of 20:50", which itself generates "Why not?" and is then followed with a brief exchange about how to book and yet another question about 3D glasses. This short sequence has many pairs of questions and answers, and indeed layers of them. This means there are other questions such as "Have you booked tickets?" or "Can you book?" that form 'insertion sequences', where a question is inserted before the answer to an original question is given [47].

Yet these questions are perhaps best seen not as ways of searching for information. Considered instead as actions, they are about arranging a cinema visit, getting responses from those who want to go and sorting out which format to go and see and when. The meaningfulness of this exchange is visible because of its sequential organisation. Hence, David's response "Why not?" is a response to going at 20:50 (not Marcus's initial offer of 17:05 to Philip), not a search for reasons not to go. David's response arrives sequentially after. The sequential order of the turn is intrinsic to what is actually said and thus is vital for understanding what is being done and communicated.

\section{Sequential context}

Staying with Example 3, we can see that David's replies do not directly refer to the previous discussion with Philip. Yet they do set a sequential context for the conversation, and if they were switched around this would cause some confusion. David, for example, assumes that they will be going to see the film in 3D (and mentions that they already have glasses). Here, the prior turns work to set the sequential context for the subsequent discussion: prior turns give context. As Manyard puts it "An utterance's context is the organized sequence of turns in which it appears" [32 p. 64]. "Thinking of 20:50" on its own is ambiguous, but it collects its context from prior turns that are mutually available to reader and writer. "20:50" is possibly a time, and a time that is visibly relevant as a start time for the movie "Pacific Rim", playing today.

This might seem an obscure point were it not for the fact that sequentiality is something that can break down during online conversations. For instance, one of our participants related a story about the deletion of a spam comment on a photo posted on Instagram. Its deletion broke sequentiality, and her 'put down' (which referred to the spam) was taken to be referring to the original photo, resulting in another user 'calling her out' and the subsequent need for explanation. Indeed, sequentiality and its relationship to maintaining context turns out to be more broadly problematic in social media than it is in spoken conversation, because systems can disrupt or change sequences in ways that would be impossible in talk.

So, in social media systems a simple 'oldest first' rule is most commonly used to order contributions. This form preserves sequentiality in most cases (although with some issues around simultaneous composition-duplicate posts). Yet it offers little priority to highlight more interesting contributions. It is for this reason that high traffic sites such as Slashdot and Reddit have pioneered user voting systems to establish this sense of priority. These sites rely upon 'replies' being hierarchically separated, so that two comments to the same prior turn are separate from each other and may be positioned in different ways. This means that while sequentiality can be preserved through replies, sequentiality between multiple comments is sacrificed (at this point we note that Facebook has recently implemented elements of threading via direct replies to comments on a shared post). Although such sites rearrange contributions, meaning that regular contributors quickly learn how to make use of the different sequences generated, this is not to say that confusion is entirely avoided. This said - and leaving aside for a moment attempts to design systems that address this (e.g., Smith et al. [53], and Viegas and Donath [57]) - most analytic work around threaded conversation has ignored the role of sequentiality and focused on aggregate relationships between threaded items. For 
instance, Weninger et al. look at the topical agreement in comment threads, finding that the aggregate comments in discussion threads are topically similar to one another [61]. What is missing here is analysis of attempts to preserve sequence itself and whether-and how-users manage the sequential presentation.

\section{Turn allocation and speaker selection}

One of the most basic contributions of EMCA has been in detailing how conversationalists practically manage the basic problem of not talking at the same time: whose turn it is to speak and when. EMCA argues that this 'problem' is managed in and through utterances themselves. In their classic foundational paper for conversation analysis, Sacks et al. point to two major genres of speaker selection for the allocation of turns: "current speaker selects next" and "selfselection" [45]. For dyadic and multiparty conversations, turn allocation methods are distributed into these two forms. Self-selection occurs simply when a speaker takes the next turn in a conversation having not been selected in some way (see Example 4, bottom left, where a user selfselects with "Hey long time no see!"). For the "current speaker selects next" form, various turn-allocation techniques may be employed, such as the use of first pairparts (such as a question, as discussed above) or pre- or post-positioned names [26]—-such as "Mary, how are you?" or "How are you, Mary?". Methods of turn allocation also "set constraints" on what can be done by a subsequent speaker [45].

Given the foundational nature of turn allocation methods in talk, it is perhaps no surprise that the question of 'whose turn' is managed in social media environments in similar ways. One first, trivial observation here is that status updates or tweets are themselves often a case of speaker self-selection. But beyond this how are subsequent 'responses' to these produced? One way to do this is through '@-formulations' of other users' names. Social media systems have become relatively standardised on this @-formulation, either as matter of explicit syntactic form (as in '@user') or as a result of merely typing another user's name (e.g., Facebook). Doing @-formulations typically results in a notification or alert to a given user that their name was 'mentioned'. This formulation-along with the "spatial relations" of the page [9]—provides resources for users to manage turn allocation. In many ways this @formulation mirrors that of pre- and post-positioned naming associated with first pair-parts in talk, yet has subtle differences. For instance, Lerner argues that post-positioned names (e.g., "How are you Mary") can be a "device to demonstrate a particular stance toward or relationship with a recipient" such as to "underscore personal concern for a problem" [26], whereas it is not clear that the @formulation does similar work on social media. However, use of the@-formulation does nevertheless provide for a range of turn allocation techniques. Consider the three Instagram screenshots shown in Example 4. At the top of Example 5, the user posting a photo ("che") mentions two other users, one of whom ("ren") subsequently posts "Haha I love it". While this may look like the @-formulation is acting as the first pair-part of a summons-answer, the user responding orients to the photo itself (not fully shown).

In the bottom left of Example 4, we see an instance of the @--formulation being used in a pre-positioned way before a second pair-part, i.e., “@riri hahah” in response to riri’s "Hey long time no see!" which its itself a sequentially relevant response to the photo posting. Finally on the bottom right of Example 5, the @-formulation is postpositioned as part of a question posed by jimi, "OMG who is this@classy" directed at the user posting the original photo-the user "classy" then disambiguates an answer to the two questions posted (i.e., "OMG who is this" and "Why don't I know this girl?") by also using the @formulation in spite of the sequential implication of posting directly beneath-i.e., classy's use of @tickn displays an orientation to the potential for sequential breakdowns as we described earlier.

Our point here is that @-formulations can be deployed as a way of managing the fact that 'anyone' is potentially "in play" [46] on social media, while at the same time inviting a response. In this sense it is not really about constructing 'presence' as one might assume. This is likely due to social media system design routinely favouring multiparty communication (e.g., status updates to friends on Facebook, tweets to followers on Twitter, etc., although note our earlier caveat on Facebook's direct reply features). Management of this environment can be achieved by 'listing' multiple others, as we see Example 4 (top).

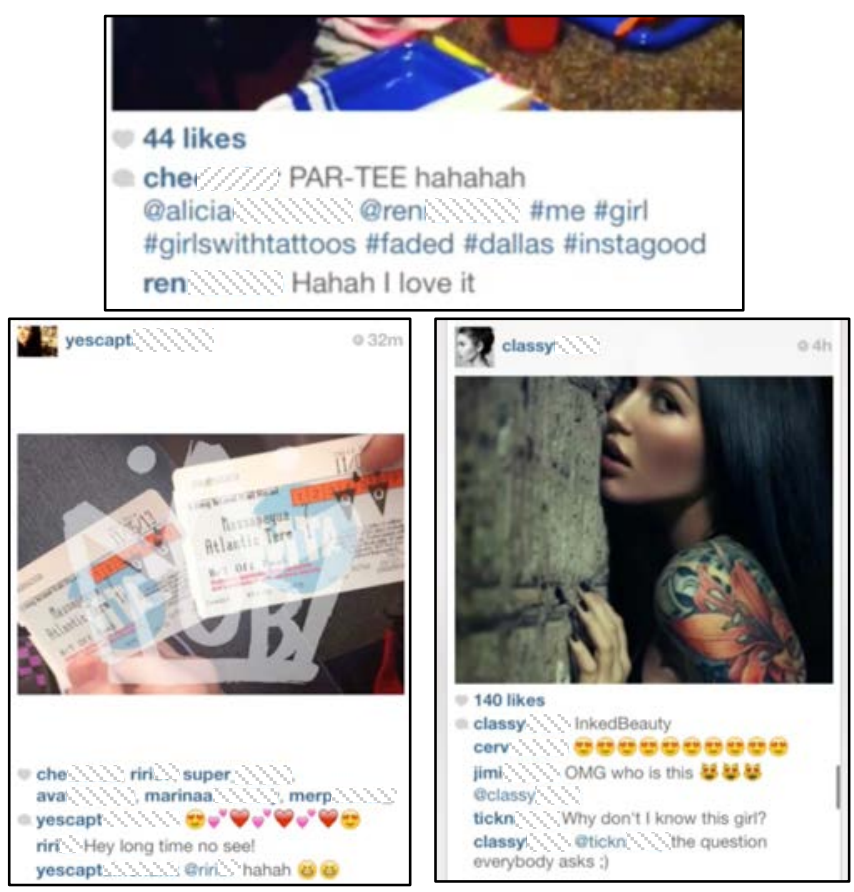

Example 4: @-formulations as speaker selection 
This does various bits of work for the user: it invites a response, frames the sense of subsequent posts, and also constrains what may actions be done in subsequent posts by selected speakers (e.g., 'appreciation'). We also note here that the@-formulation can of course also be used to preserve sequentiality through speaker selection, as we pointed to in Example 5 (bottom right), where the user deploys it for their answer but in a way that indicates it is part of a sequence with a particular user.

\section{Repair}

The fourth sequential feature that we will discuss is repair. Repair in conversation is a pervasive feature of talk in that participants will fix previous utterances that are unclear, ambiguous or wrong in some locally-determined way. Since in talk we cannot go back in time and 'edit' what has previously been said, subsequent turns are often used by speakers-but also by co-conversationalists - to correct what is said. A simple example can illustrate from [49]:

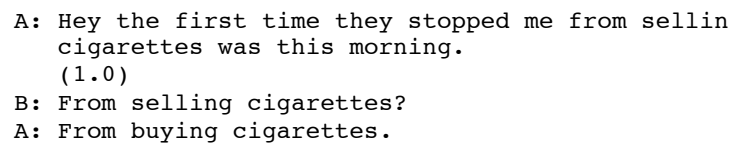

Here the speaker confuses buying and selling cigarettes. The second speaker than initiates repair by questioning the "selling cigarettes", which then is repaired by the original speaker. Repair types vary, but broadly can be self- or other-repair, each of which may be, in turn, self- or otherinitiated. The example above is an other-initiated self-repair in that the speaker (A) performs the repair, but it is the other (B) who initiates it by producing a question first pairpart ("From selling cigarettes?"). But how might methods of repair feature in social media use? There has been some work examining this; for instance, Meredith and Stokoe analyse repair in Facebook text chat communication, focusing on self-initiated repair [34]. Repairs can come in the next turn after a completed entry after, such as in Example 5. Here a 'repair' comes after the initial comment post, changing "you live?" to "you live!". This is a selfinitiated self-repair.

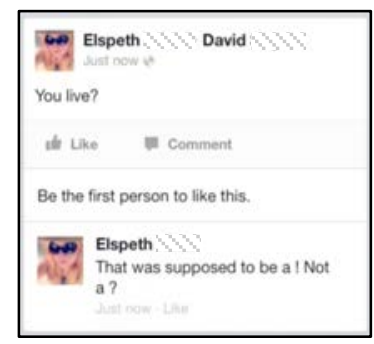

Example 5: You live?

However, we would suggest that repair is less common in social media precisely because it is, to an extent, possible to go back in time and 'edit' what one has said. While composing a message an author can go back and correct mistakes or typographic errors. An important point to take from the EMCA notion of repair is that it is an interactional and collaboratively-achieved phenomenon. Since edits are private and not available to others, this does not count as repair (unless the editing is shared locally). We can see a counterpoint to this in Brown and Bell's study of the 'There' game / chat environment. In that system, text typed by users is shared in-the-moment, word by word as it is typed (in speech bubbles above an avatars' heads) [5]. In that case participants can attempt repair of turns as they are produced by other speakers.

\section{DISCUSSION}

Through examining social media in a way informed by ethnomethodology and conversation analysis, we have sought to highlight its potential as a complementary and generative perspective alongside existing ways of studying social media. In this paper we have offered two key ways to think about social media. Firstly, we explored the momentby-moment use of social media in the life of its users, and how it is practically arranged and embedded in their everyday activities and engagements. Secondly, we have described how the concept of sequentiality-and related sequential structures - can be used to understand the organisation of social media use.

But how might this focus on lived experience be a productive activity for social media research? EMCA supports an unrelentingly close focus on the 'lived reality' of social media use. If we were to criticise the aggregate and actor-focussed perspectives at all, then it would be to say that they tend to miss certain phenomena that become observable only when we examine the details of how interactions on social media systems are not only made orderly (e.g., via adjacency pairs, sequential context, turn allocation / speaker selection, and repair), but also how those interactions are themselves embedded in the broader orderings of everyday life (e.g., how meaningful interactions around the posting of "Whee!" were not just 'located' on Facebook). Key to this order is the notion of it forming in real time-moment-by-moment for those involved. This is an order that is always partially assembled in the perceived world as it unfolds. Moreover this is an order that is recognisable and observably oriented to by those involved; one that can be found by looking at social media use as it takes place. It is an order that is itself discovered and produced by those using this 'new' thing (social media technologies). In this sense social media use is a joint endeavour that involves creating new order structures seen in common between users. Hence, it is more fitting to suggest that the EMCA approach is interactionand order-focussed rather than actor-focussed. To put this another way, since we all need to work together in various ways (such as in talk), action is arranged so that it is understandable to our co-participants, for all practical purposes. This means looking at actual action and activity, avoiding a priori methods of mass categorisation, and instead examining participants' deployments of categories over the analyst's. 
As an approach, EMCA clearly has various limitations for the study of social media use; here we must separate comments regarding the EMCA perspective and the particular approach to data collection we employed. On the first topic, we note that EMCA perspectives do not answer questions about broad behavioural trends or address network properties that may be uncovered by aggregate perspectives. Equally, it does not directly elicit prospective user needs for design that may be drawn out (e.g., in interviews) through actor-focussed perspectives. Further, the reliance on examining social order closely often results in very intensive forms of data collection that require lengthy periods of analysis of complex, heterogenous and ultimately extremely rich data sets. Finally, and turning to the second topic, in our servicing of the EMCA concern for preserving sequential context via our use of rich audiovisual data, we acknowledge that the particular user-driven data collection technique reported in this paper may present potential ethical challenges regarding establishing the consent of third parties to the (consenting) participants.

Our second point for this discussion is how the EMCA approach interacts with the design of social media systems. Historically, EMCA's role in CSCW has been broadly productive in terms of design (although we note this has not been without tensions [52]). By focussing on the momentby-moment use of social media and how it is embedded in everyday life in very practical ways, the perspective here draws attention to particular design elements that are immediately under the control of technologists - such as when content is posted and sequentially what is associated with it. One extreme example of a break down in sequentiality is found in prior studies of IRC [38]. The '@' formulation is an attempt to remedy this by providing resources to retain sequentiality to support the production of adjacency pairs in a situation where it might not be possible to produce a second pair-part sequentially after its first pairpart, as we saw with photo posting on Instagram in particular. Interestingly, this ' $@$ ' formulation makes use of a very established speaker selection method from talk [26]. We also note that the design of Facebook's commenting mechanism has recently introduced greater support for sequentiality via direct replies to comments (whereas before these were temporally organised only). Of course users also find their own methods for managing sequentiality (e.g., SMS [22], IRC [38, 21]).

Here we might return to our example of threaded chat systems such as adaptations of IRC-like environments (e.g., [53]) or web forums that attempt to support more threaded conversations. Yet these systems tend to 'hide' chat exchanges away and thus reduce the opportunities created by the idea that 'anyone' is in play on social media. Threading can also inadvertently remove the "single point of focus" present in linear online chat systems [53] - an important resource for interaction. So, we might wonder then how, rather than subverting the use of the '@' formulation, we might extend it such as by seeing how we could use the notification process to communicate different forms of second pair part (such as replied, asked, called, and so on).

Building upon this we can also see that particular resources for repair are provided, again with impacts on the methods used by users to order their interactions online. For instance, we have seen how social media reduces the visibility of repair as it is done in a moment-by-moment way; instead it is largely private. Alternative designs of chat systems suggest that exposing typing in real time offers users different conversational possibilities, such as projecting the end of a sentence (aiding turn-taking and increasing the speed of exchanges), or enabling others to complete a turn or repair it for another user (which can be used to demonstrate alignment) [5].

Our points here are not offering a critique of particular designs. Instead the EMCA perspective helps us understand just how particular and quite subtle design choices can have significant impacts upon the very methods with which users go about making their interactions with social media orderly in the first place. EMCA lets us more easily see what is lost but also what is gained as these design decisions fit with the actual users' methods.

\section{CONCLUSION}

We have sought to articulate an alternative perspective for social media research. To this end we have not presented a paper involving a singular study, but rather sketched some programmatic possibilities around how EMCA approaches can enrich existing ways of studying social media use. The key contribution of this paper has been to begin scratching the surface of what the study of social media use might reveal when we examine it in terms of classic EMCA topics: of its moment-by-moment embeddedness in everyday life, and of the methods of managing the experience of that everyday life through sequential order. We have indicated some future ways in which studies could proceed by considering similarities to some of the methods of everyday talk: adjacency pairs, sequential context, turn allocation / speaker selection, and repair.

Although we see these as potentially generative investigations for social media research and design, the rich body of EMCA work also offers many more that we have not had space to cover, for example how conversational 'closings' are achieved in talk [48]. We might ask how (and whether) similar phenomena are organised for social media. There are also many features of social media systems themselves that have been left untouched here, for example Facebook's news feed could well be examined along these lines. We also are hopeful that thinking about sequentiality could let us design different social media systems. Lastly, by working through EMCA and social media we may reveal aspects of both that have thus far been neglected. 


\section{ACKNOWLEDGMENTS}

This work is supported by EPSRC (EP/K025848/1) and grants from the Swedish Governmental Agency for Innovation Systems to the Mobile Life VinnExcellence Centre, in partnership with Ericsson, Microsoft, Nokia, IKEA and the City of Stockholm. We thank Moira McGregor for collecting the data examined in this paper. We also want to acknowledge anonymous reviewers of this paper for their valuable input.

Data access statement: Due to ethical concerns and consent restrictions, supporting research data (collected by Brown) cannot be made openly available.

\section{REFERENCES}

1. Lars Backstrom and Jon Kleinberg. 2014. Romantic partnerships and the dispersion of social ties: a network analysis of relationship status on Facebook.

In Proceedings of the 17th ACM conference on Computer supported cooperative work \& social computing (CSCW '14). ACM, New York, NY, USA, 831-841.

2. Michael S. Bernstein, Eytan Bakshy, Moira Burke, and Brian Karrer. 2013. Quantifying the invisible audience in social networks. In Proc. CHI'13. ACM, New York, NY, USA, 21-30, 2013.

3. Danah Boyd. 2014. It's Complicated: The Social Lives of Networked Teens. Yale University Press.

4. Barry Brown, Moira McGregor, and Donald McMillan. 2015. Searchable Objects: Search in Everyday Conversation. In Proceedings of the 18th ACM Conference on Computer Supported Cooperative Work $\&$ Social Computing (CSCW '15). ACM, New York, NY, USA, 508-517.

5. Barry Brown and Marek Bell. 2004. CSCW at play: 'there' as a collaborative virtual environment. In Proceedings of the 2004 ACM conference on Computer supported cooperative work (CSCW '04). ACM, New York, NY, USA, 350-359.

6. Moira Burke, and Robert Kraut. 2013. Using Facebook after losing a job: differential benefits of strong and weak ties. In Proc. CSCW'13. ACM, New York, NY, USA, 1419-1430, 2013.

7. Carlos Castillo, Mohammed El-Haddad, Jürgen Pfeffer, and Matt Stempeck. 2014. Characterizing the life cycle of online news stories using social media reactions. In Proc. CSCW'14. ACM, New York, NY, USA, 211-223, 2014.

8. Emily Erikson. 2013. Formalist and Relationalist Theory in Social Network Analysis. Sociological Theory, 31(3):219-242, Sage 2013.

9. Maximiliane Frobenius, and Richard Harper. 2015. Tying in comment sections: The production of meaning and sense on Facebook. Semiotica. 2015(204): 121143.
10. Ge Gao, Pamela Hinds, and Chen Zhao. 2013. Closure vs. structural holes: how social network information and culture affect choice of collaborators. In Proc. CSCW'13. ACM, New York, NY, USA, 5-18, 2013.

11. Harold Garfinkel. 1967. Studies in ethnomethodology. Polity Press.

12. Eric Gilbert and Karrie Karahalios. 2009. Predicting tie strength with social media. In Proc. CHI'09. ACM, New York, NY, USA, 211-220, 2009.

13. Erving Goffman. 1966. Behavior in Public Places: Notes on the Social Organization of Gatherings. Free Press.

14. Jennifer Golbeck. 2013. Analyzing the Social Web. Elsevier.

15. Charles Goodwin. 1986. Gestures as a resource for the organization of mutual orientation, Semiotica 62 (12):29-50 (1986).

16. Marjorie H. Goodwin. 1998. Games of Stance: Conflict and Footing in Hopscotch. In Kids' Talk: Strategic Language Use in Later Childhood, Susan Hoyle and Carolyn Temple Adger (eds.), 23-46, New York: Oxford University Press.

17. Rebecca Gray, Nicole B. Ellison, Jessica Vitak, and Cliff Lampe. 2013. Who wants to know? Questionasking and answering practices among Facebook users. In Proc. $C S C W$ '13. ACM, New York, NY, USA, 1213-1224.

18. Christian Heath, Jon Hindmarsh, and Paul Luff. 2010. Video in qualitative research. Sage Publications.

19. Libby Hemphill and Andrew J. Roback. 2014. Tweet acts: how constituents lobby congress via Twitter. In Proc. CSCW'14, ACM, New York, NY, USA, 12001210, 2014.

20. Ai-Ju Huang, Hao-Chuan Wang, and Chien Wen Yuan. 2014. De-virtualizing social events: understanding the gap between online and offline participation for event invitations. In Proc. CSCW'14, ACM, New York, NY, USA, 436-448, 2014.

21. Ian Hutchby. 2000. Conversation and Technology: From the Telephone to the Internet (chapter 9). Polity Press.

22. Ian Hutchby and Vanita Tanna. 2008. Aspects of sequential organization in text message exchange. Discourse \& Communication, May 2008 2:143-164

23. Michal Jacovi, Ido Guy, Shiri Kremer-Davidson, Sara Porat, and Netta Aizenbud-Reshef. 2014. The perception of others: inferring reputation from social media in the enterprise. In Proc. CSCW'14.

24. Gail Jefferson. 2004. Glossary of transcript symbols with an introduction. In Gene. H Lerner (ed). Conversation Analysis: Studies from the First 
Generation, pp. 13-31. Amsterdam: John Benjamins, 2004.

25. Cliff Lampe, Rebecca Gray, Andrew T. Fiore, and Nicole Ellison. 2014. Help is on the way: patterns of responses to resource requests on Facebook. In Proc. CSCW'14. ACM, New York, NY, USA, 3-15, 2014.

26. Gene H. Lerner. 2003. Selecting next speaker: The context-sensitive operation of a context-free organization. Language in Society, 32, 177-201.

27. Jessica Lingel, Mor Naaman, and danah m. boyd. 2014. City, self, network: transnational migrants and online identity work. In Proc. CSCW'14, ACM, New York, NY, USA, 1502-1510, 2014.

28. Eden Litt, Erin Spottswood, Jeremy Birnholtz, Jeff T. Hancock, Madeline E. Smith, and Lindsay Reynolds. Awkward encounters of an "other" kind: collective self-presentation and face threat on Facebook. In Proc. $C S C W^{\prime} 14$, ACM, New York, NY, USA, 449-460, 2014.

29. Christian Licoppe and Julien Figeac. 2013. Patterns of Gaze Switching in the "Naturally-Occurring" Uses of Smartphones in Urban Mobile Settings. Department of Economics and Social Sciences, TELECOM ParisTech, 2013.

30. Michael Lynch. 1997. Scientific Practice and Ordinary Action. Cambridge University Press.

31. Michael Lynch. 2009. The ethnomethodological foundations of conversation analysis. Text Interdisciplinary Journal for the Study of Discourse, 20(4), pp. 517-532.

32. Douglas A. Maynard. 2003. Bad News, Good News: Conversational Order in Everyday Talk and Clinical Settings. The University of Chicago Press.

33. Moira McGregor, Barry Brown, and Donald McMillan. 2014. 100 days of iPhone use: mobile recording in the wild. In CHI'14 Extended Abstracts on Human Factors in Computing Systems (CHI EA '14). ACM, New York, NY, USA, 2335-2340.

34. Joanne Meredith and Elizabeth Stokoe. 2014. Repair: Comparing Facebook 'chat' with spoken interaction. Discourse and Communication, 8(2):181-207, Sage, 2014.

35. Alan Mislove, Massimiliano Marcon, Krishna P. Gummadi, Peter Druschel, and Bobby Bhattacharjee. 2007. Measurement and analysis of online social networks. In Proceedings of the 7th ACM SIGCOMM conference on Internet measurement (IMC '07). ACM, New York, NY, USA, 29-42.

36. Robert J. Moore, Elizabeth F. Churchill, and Raj Gopal Prasad Kantamneni. 2011. Three sequential positions of query repair in interactions with internet search engines. In Proceedings of the ACM 2011 conference on Computer supported cooperative work (CSCW '11). ACM, New York, NY, USA, 415-424.

37. Meredith Ringel Morris. 2014. Social networking site use by mothers of young children. In Proc. CSCW'14.

38. Jacki O'Neill and David Martin. 2003. Text Chat in Action. in Proceedings of Group '03, ACM Press, New York, 2003.

39. Anita Pomerantz. 1985. Agreeing and disagreeing with assessments: some features of preferred/dispreferred turn shapes. In J. Maxwell Atkinson (ed). Structures of Social Action, pp. 57-101. Cambridge University Press, 1985.

40. Daniele Quercia, Jonathan Ellis, Licia Capra, and Jon Crowcroft. 2012. Tracking gross community happiness from tweets. In Proc. CSCW'12. ACM, New York, NY, USA, 965-968, 2012.

41. Daniele Quercia, Renaud Lambiotte, David Stillwell, Michal Kosinski, and Jon Crowcroft. 2012. The personality of popular Facebook users. In Proc. CSCW '12. ACM, New York, NY, USA, 955-964, 2012.

42. John Rooksby, Timothy E. Smith, Alistair Morrison, Mattias Rost, and Matthew Chalmers.

2015. Configuring Attention in the Multiscreen Living Room. To appear in Proc. of ECSCW 2015, 19th-23rd September 2015, Oslo, Norway.

43. John Rooksby and Ian Sommerville. 2012. The Management and Use of Social Network Sites in a Government Department. Computer Supported Cooperative Work, 21(4-5): 397-415.

44. Harvey Sacks, Gail Jefferson, and Emanuel Schegloff. (Eds.) 1992. Lectures in Conversation. Oxford: Blackwell.

45. Harvey Sacks, Emanuel A. Schegloff, and Gail Jefferson. 1974. A Simplest Systematics for the Organization of Turn-Taking for Conversation. Language, Vol. 50, No. 4, Part 1 (Dec., 1974), pp. 696-735.

46. Emanuel A. Schegloff. 1968. Sequencing in Conversational Openings. American Anthropologist. New Series, Vol. 70, No. 6 (Dec., 1968), pp. 1075-1095.

47. Emanuel A. Schegloff. 1972. Notes on a conversational practice: formulating place. In Sudnow, D. (ed), Studies in social interaction, New York, Free Press.

48. Emanuel A. Schegloff and Harvey Sacks. 1973. Opening Up Closings. Semiotica, VIII, 4 (1973) 289327.

49. Emanuel A. Schegloff, Harvey Sacks, and Gail Jefferson. 1977. The Preference for Self-Correction in the Organization of Repair in Conversation. Language, 53, 361-382. 
50. Emanuel A. Schegloff. 2007. Sequence Organization in Interaction: A primer in conversation analysis, volume 1. Cambridge University Press.

51. Dan Shapiro. 1994. The limits of ethnography: combining social sciences for CSCW. In Proceedings of the 1994 ACM conference on Computer supported cooperative work (CSCW '94). ACM, New York, NY, USA, 417-428.

52. Wes Sharrock and Dave Randall. 2004. Ethnography, ethnomethodology and the problem of generalisation in design. European Journal of Information Systems 13, 186-194 (1 September 2004).

53. Marc Smith, J. J. Cadiz, and Byron Burkhalter. 2000. Conversation trees and threaded chats. In Proceedings of the 2000 ACM conference on Computer supported cooperative work (CSCW '00). ACM, New York, NY, USA, 97-105.

54. Tasos Spiliotopoulos and Ian Oakley. 2013. Understanding Motivations for Facebook Use: Usage Metrics, Network Structure, and Privacy. In Proc. CHI'13, Paris, 2013.

55. Yuri Takhteyev, Anatoliy Gruzd, and Barry Wellman. 2012. Geography of Twitter networks. Social Networks, 34(1):73-81, January 2012.

56. Sherry Turkle. 2011. Alone together. Basic Books.

57. Fernanda B. Viégas and Judith S. Donath. 1999. Chat circles. In Proceedings of the SIGCHI conference on Human Factors in Computing Systems (CHI '99). ACM, New York, NY, USA, 9-16.

58. Jessica Vitak and Jinyoung Kim. 2014. "You can't block people offline": examining how Facebook's affordances shape the disclosure process. In Proc. CSCW '14. ACM, New York, NY, USA, 461-474.

59. Duncan J. Watts. 1999. Networks, Dynamics and the Small-World Phenomenon. American Journal of Sociology, 105(2):493-527, 1999.

60. Alexandra Weilenmann, Daniel Normark, and Eric Laurier. 2014. Managing Walking Together: The Challenge of Revolving Doors. Space and Culture, 17(2), 122-136.

61. Tim Weninger, Xihao Avi Zhu, and Jiawei Han. 2013. An exploration of discussion threads in social news sites: a case study of the Reddit community. In Proceedings of the 2013 IEEE/ACM International Conference on Advances in Social Networks Analysis and Mining (ASONAM '13). ACM, New York, NY, USA, 579-583.

62. Hui Zhang, Munmun De Choudhury, and Jonathan Grudin. 2014. Creepy but inevitable?: the evolution of social networking. In Proc. CSCW'14, ACM, New York, NY, USA, 368-378, 2014. 